\title{
QUALIDADE DE VIDA, FUNCIONALIDADE E DOR EM MULHERES JOVENS COM DOR PATELOFEMORAL
}

\author{
QUALITY OF LIFE, FUNCTIONALITY AND PAIN IN \\ YOUNG WOMEN WITH PATELLOFEMORAL PAIN
}

Amanda Paula Ricardo (Orcid: 0000-0002-9687-5690)

Camile Ludovico Zamboti (Orcid: 0000-0001-7283-4934) ${ }^{1}$

Gabriela de Paula (Orcid: 0000-0002-5987-7400) ${ }^{2}$

Christiane de Souza Guerino Macedo (Orcid: 0000-0001-6016-5075) ${ }^{1}$

\section{Contato}

Christiane de Souza Guerino Macedo E-mail: chmacedouel@yahoo.com.br

${ }^{1}$ Programa de Pós-Graduação em Ciências da Reabilitação, Universidade Estadual de Londrina/Universidade do Norte do Paraná, Paraná, Brasil.

${ }^{2}$ Curso de Fisioterapia, Universidade Estadual de Londrina, Paraná, Brasil.

\section{RESUMO}

Objetivo: verificar a influência da síndrome da dor patelofemoral (SDPF) na qualidade de vida, funcionalidade e dor de mulheres jovens. Métodos: a amostra foi composta por 20 participantes, com idades entre 19 e 24 anos, distribuídas em grupo SDPF $(\mathrm{N}=10)$ e controle $(\mathrm{N}=10)$. Estas responderam ao questionário Medical Outcome Study 36-item Short Form (SF36) de qualidade de vida, Anterior Knee Pain Scale (AKPS) para análise da funcionalidade e escala visual analógica (EVA). Para análise estatística, utilizaram-se os testes Shapiro Wilk, t Student e Correlação de Pearson, com o SPSS ${ }^{\circledR}$ 20. Resultados: no grupo com SDPF, encontrou-se pior qualidade de vida, em específico, para capacidade funcional $(\mathrm{p}<0,001)$, aspectos físicos $(\mathrm{p}=0,005)$, dor $(\mathrm{p}<0,001)$ e aspectos emocionais $(\mathrm{p}=0,031)$ do questionário SF-36. Também houve diferenças de funcionalidade $(\mathrm{p}<0,001)$ e dor $(\mathrm{p}<0,001)$ avaliadas em mulheres jovens. No grupo SDPF, foi estabelecida forte correlação entre funcionalidade (AKPS) e capacidade física $(r=0,874)$, dor $(r=0,771)$, vitalidade $(r=0,771)$ e saúde mental $(r=0,743)$. Para o grupo controle, a AKPS apresentou forte correlação somente com o estado geral de saúde $(r=0,715)$. Conclusão: mulheres jovens com SDPF apresentam menor qualidade de vida, tanto para os domínios físicos como para os emocionais; pior funcionalidade e maior dor. Estes fatores devem ser considerados na avaliação e reabilitação de mulheres com SDPF, incluindo as mais jovens.

Palavras-chave: Síndrome da dor Patelofemoral; Joelho; Qualidade de vida; Dor; Fisioterapia.

\begin{abstract}
Objective: to verify the influence of patellofemoral pain syndrome (PFPS) on the quality of life, functionality, and pain in young women. Methods: the sample was composed of 20 participants, aged between 19 and 24 years, allocated to the PFPS group $(n=10)$ and control group $(n=10)$. The participants answered the Outcomes Study 36-item Short Form questionnaire (SF-36) for quality of life, the Anterior Knee Pain Scale (AKPS) to analyze functionality, and the visual analog scale (VAS). The statistic analysis used the Shapiro Wilk, Student-t, and the Pearson correlation coefficient tests, using the software SPSS 20. Results: in the group with PFPS found worse quality of life, specifically capacity $(\mathrm{p}<0.001)$, physical aspects $(\mathrm{p}=0.005)$, pain $(\mathrm{p}<0.001)$, and emotional aspects $(\mathrm{p}=0.031)$ on the SF-36 questionnaire. In addition, differences in functionality $(\mathrm{p}<0.001)$ and pain $(\mathrm{p}<0.001)$ were established in young females. In the PFPS group, a strong correlation was found between functionality (AKPS), physical capacity $(\mathrm{r}=0.874)$, pain $(0.771)$, vitality $(\mathrm{r}=0.771)$, and mental health $(\mathrm{r}=0.743)$. For the control group, the AKPS presented a strong correlation only with general health state $(r=0.715)$. Conclusion: young women with PFPS presented worse quality of life, both for physical aspects and emotional aspects, worse functionality, and greater pain. These factors should be considered in the evaluation and rehabilitation of women with PFPS, including young females. Keywords: Patellofemoral pain syndrome; Knee; Quality of Life; Pain; Physiotherapy.
\end{abstract}




\section{INTRODUÇÃO}

A síndrome da dor patelofemoral (SDPF) é caracterizada por dor anterior no joelho, edema, crepitação retropatelar e bloqueio articular; agrava-se durante atividades de subida e descida de escadas, caminhadas em terrenos inclinados, agachamento e ao permanecer sentado por tempo prolongado ${ }^{1}$. A SDPF se tornou foco de pesquisas pela sua alta incidência e pela tendência em se tornar persistente e agravada em longo prazo. A dor está relacionada com a alteração do desempenho e da velocidade para a realização de tarefas diárias, bem como com a prática de atividades físicas, com consequente diminuição de qualidade de vida ${ }^{2}$. Dessa forma, instrumentos que possibilitam avaliação, quantitativa ou qualitativa, do impacto da SDPF na qualidade de vida e funcionalidade se tornaram cada vez mais importantes ${ }^{3,4}$.

A fim de avaliar a qualidade de vida, estudos têm utilizado questionários com o poder de elucidar questões geralmente não relatadas pelos pacientes, o que possibilita a avaliação objetiva dos fatores subjetivos do indivíduo ${ }^{5}$. O Medical Outcome Study 36-item Short Form (SF-36) é validado e reprodutível para diferentes populações, e sensível à melhora do indivíduo $^{6,7}$. Apesar de não ter validade específica na SDPF, foi utilizado por outros estudos como forma de avaliação da síndrome, além de ser utilizado em mais de 200 doenças e traduzido em 40 países $^{8}$.

Por outro lado, questionários específicos para SDPF possibilitam maior sensibilidade para compreensão de mu- danças sutis dos sinais e sintomas relacionados com as características da síndrome e, assim, maior compreensão das alterações e do impacto da disfunção na qualidade de vida e funcionalidade. Para avaliação da funcionalidade na SDPF, a Escala de Kujala ou Anterior Knee Pain Scale (AKPS) é amplamente utilizada ${ }^{9,10}$, possui propriedades métricas adequadas estabelecidas por Aquino et al. ${ }^{11}$, com boa confiabilidade teste e reteste e consistência interna, além de se mostrar sensível à sintomatologia da síndrome. Entretanto, o uso de escalas e questionários no dia a dia dos profissionais da saúde ainda é pouco frequente e deve ser incentivado.

Assim, em função da alta incidência da SDPF, de sua repercussão nas atividades de vida diárias e da necessidade de avaliar de forma objetiva os relatos das pacientes, este trabalho visou apontar as alterações da qualidade de vida, funcionalidade e dor em mulheres jovens com SDPF. Em adição, o presente estudo correlacionou essas escalas para estabelecer se há necessidade do uso associado delas. Acredita-se que os resultados poderão justificar a importância da avaliação da qualidade de vida e funcionalidade em mulheres com SDPF.

\section{MATERIAIS E MÉTODOS}

Estudo observacional com 20 participantes do gênero feminino, selecionadas por uma amostra de conveniência, distribuídas em dois grupos: SDPF $(n=10)$ e grupo controle $(n=10)$. Para o Grupo SDPF, as participantes apresen- 
tavam dor anterior no joelho de três ou mais na Escala Visual Analógica (EVA) por um período mínimo de oito semanas antes da avaliação. Os critérios adicionais de inclusão foram: dor anterior ou retropatelar do joelho, durante pelo menos três das seguintes atividades: subir/descer escadas, agachar, correr, ajoelhar, sentar por tempo prolongado e um início insidioso dos sintomas sem relação com trauma. Para o grupo controle, foi estabelecida a ausência de dor na articulação do joelho ou em qualquer outro local dos membros inferiores nos últimos seis meses. Como critério de não inclusão, para ambos os grupos, foi considerada a presença de história de lesão no sistema osteomioarticular do quadril, joelho e tornozelo; presença de doenças neurológicas, cardiovasculares ou reumatológicas; voluntárias diagnosticadas com diabetes ou alteração de sensibilidade na face plantar; e uso de medicação e/ou fisioterapia prévia ao estudo. Caso a participante se recusasse a participar do estudo e a responder ao questionário, bem como se não apresentasse capacidade cognitiva para responder às perguntas, seria excluída do estudo.

As voluntárias preencheram o questionário de Qualidade de Vida (SF36), responderam às Escalas AKPS e EVA com relação à dor no joelho. A EVA, é um instrumento unidimensional para a avaliação da intensidade da dor consiste em uma escala de $10 \mathrm{~cm}$, na qual as extremidades definem o mínimo e máximo da percepção da dor ${ }^{12}$. A Escala AKPS, foi criada por Kujala ${ }^{13}$ e foi traduzida e adaptada em vários países, inclusive no Brasil $^{14}$. Os itens avaliados na AKPS são: dor anterior no joelho, limitações funcionais durante as atividades diárias como subir escadas e caminhar, bem como aspectos como a história de subluxação patelar, claudicação na marcha e a presença da dor. A pontuação varia de zero a 100 pontos, em que 100 significa sem dores e/ou limitações funcionais e zero significa dor constante e limitações funcionais. O questionário SF-36 é um instrumento genérico de avaliação de qualidade de vida, de fácil administração e compreensão. É um questionário multidimensional formado por 36 itens, englobados em 8 escalas ou componentes: capacidade funcional, aspectos físicos, dor, estado geral da saúde, vitalidade, aspectos sociais, aspectos emocionais e saúde mental. Apresenta um escore final de 0 a 100, no qual zero corresponde a pior estado geral de saúde, e 100, a melhor estado de saúde ${ }^{15,16}$. Ambos os questionários foram autoaplicados; e, caso as voluntárias tivessem dúvidas, era realizada explicação da questão.

A análise estatística utilizou o teste Shapiro Wilk para avaliação da distribuição dos dados; e por se tratar de dados com distribuição normal, aplicouse $o$ teste $t$ Student para a comparação. Por fim, analisou-se a correlação entre as Escalas AKPS e EVA e os domínios do questionário SF-36 de qualidade de vida, 
por meio do teste correlação de Pearson. O software utilizado para as análises foi o SPSS 22 , e o nível de significância foi estabelecido em $5 \%$.

A pesquisa foi conduzida de acordo com a Resolução 466/2012 do Conselho Nacional de Saúde, iniciada após a aprovação do Comitê de Ética em Pesquisa da Universidade Estadual de Londrina (Parecer: 1.024.565). Todas as participantes foram esclarecidas sobre os procedimentos do estudo e assinaram o Termo de Consentimento Livre e Esclarecido com informações a respeito dos objetivos do estudo e questionários utilizados.

\section{RESULTADOS}

Os resultados de caracterização da amostra estão apresentados na Tabela 1; em relação aos dados antropométricos, observaram-se grupos homogêneos em relação à idade, peso e altura. Quando analisados os resultados das escalas e questionários (Tabela 2), verificou-se que o grupo SDPF apresentou piores escores de qualidade de vida (capacidade funcional, aspectos físicos, dor e aspectos emocionais), funcionalidade e dor.

Tabela1. Caracterização da amostra. Resultados apresentados em média e desvio padrão.

\begin{tabular}{|c|c|c|c|}
\hline Grupos & Idade (anos) & Peso (Kg) & Altura (m) \\
\hline Grupo com dor & $21,2 \pm 2,18$ & $69,9 \pm 12,14$ & $1,66 \pm 0,08$ \\
\hline Grupo controle & $22,1 \pm 2,17$ & $60,0 \pm 11,67$ & $1,61 \pm 0,06$ \\
\hline$p^{\dagger}$ & 0,391 & 0,095 & 0,169 \\
\hline
\end{tabular}

${ }^{\dagger}$ Teste T de Student

Tabela 2. Resultados dos questionários apresentado em média e desvio.

\begin{tabular}{lccc}
\hline \multicolumn{1}{c}{ Desfechos analisados } & Grupo com dor & Grupo controle & $\boldsymbol{p}^{\dagger}$ \\
\hline AKPS & $66,70 \pm 12,66$ & $99,80 \pm 0,63$ & $<0,001$ \\
\hline EVA & $3,90 \pm 2,02$ & $0,00 \pm 0,00$ & $<0,001$ \\
\hline SF-36 & & \\
\hline Capacidade Funcional & $63,0 \pm 20,57$ & $97,00 \pm 5,67$ & $<0,001$ \\
Aspectos Físicos & $65,00 \pm 37,63$ & $100,00 \pm 0,00$ & 0,005 \\
Dor & $50,30 \pm 20,78$ & $85,60 \pm 9,22$ & $<0,001$ \\
Estado Geral de Saúde & $63,30 \pm 19,47$ & $71,10 \pm 16,7$ & 0,381 \\
Vitalidade & $48,00 \pm 20,47$ & $54,50 \pm 20,33$ & 0,492 \\
Aspectos Sociais & $72,45 \pm 33,71$ & $83,70 \pm 23,71$ & 0,415 \\
Aspectos Emocionais & $46,60 \pm 50,15$ & $93,20 \pm 14,33$ & 0,031 \\
Saúde Mental & $57,60 \pm 17,90$ & $69,60 \pm 22,32$ & 0,068 \\
\hline
\end{tabular}

${ }^{\dagger}$ Teste $\mathrm{T}$ de Student; AKPS= Anterior knee pain scale; EVA= Escala visual analógica. 
A análise da correlação entre as Escalas EVA, AKPS e os parâmetros do questionário SF-36 para o grupo com dor patelofemoral foi considerada forte entre AKPS e capacidade física, dor, vitalidade e saúde mental; e moderada entre EVA e capacidade física, aspectos físicos, estado geral de saúde, vitalidade, aspectos sociais e saúde mental. Para o grupo controle, com relação à pontuação da AKPS, observou-se forte correlação com o estado geral de saúde, moderada com o domínio dor, e as demais correlações foram fracas (Tabela 3). Como a dor na EVA dessas participantes foi igual a zero, não foi possível realizar a correlação da EVA com os domínios do SF-36 e AKPS.

\section{DISCUSSÃO}

Por meio dos resultados encontrados, confirmou-se que mulheres jovens com SDPF apresentam menores índices de qualidade de vida, menor funcionalidade e maior intensidade de dor. Ainda, a funcionalidade avaliada pela AKPS obteve forte correlação com a capacidade física, dor, vitalidade e saúde mental em mulheres com SDPF, diferentemente do encontrado em mulheres saudáveis com forte correlação apenas da pontuação do AKPS e o estado geral de saúde.

A análise da qualidade de vida, por meio do SF-36, mostrou que os domínios, capacidade funcional, aspectos

Tabela 3. Análise das correlações entre AKPS, EVA e os domínios do questionário de qualidade de vida SF-36

\begin{tabular}{|c|c|c|c|c|c|c|}
\hline \multirow{3}{*}{ SF-36 } & \multicolumn{4}{|c|}{ Grupo com dor } & \multirow{2}{*}{\multicolumn{2}{|c|}{$\begin{array}{c}\text { Controle } \\
\text { AKPS }\end{array}$}} \\
\hline & \multicolumn{2}{|c|}{ AKPS } & \multicolumn{2}{|r|}{ EVA } & & \\
\hline & $\mathrm{r}$ & $p$ & $\mathrm{r}$ & $p$ & $\mathrm{r}$ & $p$ \\
\hline Capacidade física & 0,874 & $0,001^{*}$ & $-0,525$ & 0,119 & $-0,196$ & 0,587 \\
\hline Aspectos físicos & 0,517 & 0,126 & $-0,416$ & 0,232 & - & - \\
\hline Dor & 0,771 & $0,009^{*}$ & $-0,327$ & 0,510 & 0,610 & 0,867 \\
\hline Estado geral de saúde & 0,608 & 0,062 & $-0,456$ & 0,186 & 0,715 & $0,020^{*}$ \\
\hline Vitalidade & 0,752 & $0,012^{\star}$ & $-0,432$ & 0,212 & 0,164 & 0,650 \\
\hline Aspectos sociais & 0,582 & 0,077 & $-0,423$ & 0,224 & $-0,241$ & 0,501 \\
\hline Aspectos emocionais & 0,456 & 0,186 & $-0,133$ & 0,715 & $-0,167$ & 0,645 \\
\hline Saúde mental & 0,743 & $0,014^{*}$ & $-0,547$ & 0,102 & $-0,164$ & 0,651 \\
\hline
\end{tabular}

AKPS: Anterior Knee Pain Scale; EVA: Escala visual analógica; r: coeficiente de correlação de Pearson; * estatisticamente significativo 
físicos, dor e aspectos emocionais foram significativamente piores em mulheres jovens com SDPF. Além disso, os domínios do SF-36 avaliam principalmente o desempenho em tarefas físicas, a sensação de desânimo e a falta de energia, que são sintomas frequentes em pacientes portadores de doenças crônicas ${ }^{17}$; o que corrobora os achados na literatura ${ }^{18}$ que encontraram escores significativamente mais baixos na qualidade de vida em indivíduos com doenças crônicas musculoesqueléticas, com diferença em todos os domínios do SF-36, especialmente para a capacidade funcional, limitação por aspectos físicos e dor, mesmos domínios destacados com diferenças no presente estudo que analisou mulheres jovens com SDPF. Em adição, para destacar a importância do estudo da qualidade de vida em pacientes com SDPF, Coburn et al. ${ }^{19}$ apresentaram a diminuição nos scores em todos os domínios do SF-36. Ainda, Waiteman et al. ${ }^{20}$ descreveram a relação entre a gravidade da SDPF com a qualidade de vida de adolescentes atletas, e confirmaram que a piora dos sintomas está diretamente relacionada com a diminuição da qualidade de vida.

Em relação à incapacidade no grupo SDPF deste estudo, a pontuação estabelecida pela AKPS foi de 72,64, que é caracterizada como presença de alteração funcional relacionada com a síndrome já que está inferior a 86 pontos $^{21}$, o que confirma os achados na literatura ${ }^{22-24}$. As participantes destacaram, por meio da AKPS, que a dor patelofemoral interferiu no relacionamento familiar, profissional e social, e que as mulheres avaliadas diminuíram a realização de atividades sociais. Estes achados reforçam a ideia de que a dor patelofemoral e sua incapacidade podem influenciar e estar associadas aos parâmetros da qualidade de vida.

Nesse sentido, nossos resultados confirmam a relação entre a incapacidade funcional avaliada por meio da AKPS e a qualidade de vida avaliada com o SF-36, pois estabeleceram forte correlação entre o resultado da AKPS com os domínios capacidade física, dor, vitalidade e saúde mental, e forte correlação da dor com os aspectos sociais, a saúde mental e a vitalidade de mulheres jovens com SDPF. Corroborando os resultados do presente estudo, Cheung et al. ${ }^{25}$ encontraram correlação inversa do aumento de dor com saúde mental, função e qualidade de vida em atletas amadores e profissionais com sintomas de SDPF, e Pimenta et al. ${ }^{26}$ destacaram que a manutenção da capacidade funcional está diretamente associada à qualidade de vida. Ademais, Assa et al. ${ }^{27}$ encontraram pior capacidade funcional, com pontuação média de 33 para as mulheres. Portanto, com base nesses achados, comprova-se o impacto da incapacidade funcional na qualidade de vida de mulheres com SDPF.

Os resultados do presente estudo, portanto, sugerem a utilização do questionário SF-36 para uma avaliação mais ampla de mulheres jovens com SDPF, uma vez que inclui variáveis como aspectos emocionais e saúde mental, influenciados pela síndrome e não avaliados pela AKPS. Como limitações deste estudo, citam-se o baixo número amostral e a avaliação ape- 
nas subjetiva por meio de questionários e escalas. Para futuros estudos, sugere-se a aplicação de instrumentos de mensuração objetiva, como testes funcionais, um maior número amostral e a consideração de aspectos de sensibilidade do SF-36 pré e pós-tratamento.

\section{CONCLUSÃO}

O presente estudo observou que mulheres com SDPF apresentam pior qualidade de vida nos domínios físicos, emocionais e sociais, pior funcionalidade e maior dor quando comparadas ao grupo controle. Dentre os aspectos avaliados pelo SF-36, destacaram-se o domínio emocional e social, o que ressalta a relação deles com a SDPF, apesar de essa síndrome estar relacionada com as alterações físicas. Assim, fazem-se necessários mais estudos que avaliem o impacto de componentes emocionais e sociais na qualidade de vida de pacientes com SDPF, e a inclusão dessas abordagens na avaliação e reabilitação dessas pacientes. Por fim, a forte correlação entre os domínios do SF-36 e a AKPS estabelecem que o profissional da saúde possa optar por um deles.

\section{REFERÊNCIAS}

1. Glaviano NR, Saliba S. Impairment Based Rehabilitation for Patellofemoral Pain Patients. Phys Sportsmed. 2016; 44(3):311-323.

2. Duarte JP, Campos RS. Physical therapy to improve the quality of life in patients with patellofemoral pain syndrome. Rev Eletr Saúde Ciên 2013; $3(1): 8-22$.

3. Dutton M. Orthopaedic: Examination, evaluation, and intervention. 2nd ed. New York: McGraw-Hill; 2008.

4. Nunes GS, Stapait EL, Kirsten MH, Noronha M, Santos GM. Clinical test for diagnosis of patellofemoral pain syndrome: Systematic review with meta-analysis. Phys Ther Sport. 2013; 14(1):54-59.

5. Kawakami L, Martins ALP, Jamami M, Costa D. Avaliação dos fatores de co-morbidade e sua relação com qualidade de vida em pacientes portadores de doença pulmonar obstrutiva crônica. Rev Bras Fisioter 2005; 9(2):145-150.

6. Gonçalves FDP, Marinho PEM, Maciel MA, Galindo Filho VC, Dornelas de Andrade A. Avaliação da qualidade de vida pós-cirurgia cardíaca na fase I da reabilitação através do questionário MOS SF-36. Rev Bras Fisioter 2006; 10(1):121-126.

7. Takiut ME, Hueb W, Hiscock SB, Nogueira CRSR, Girardi P, Favarato D, et al. Qualidade de vida após revascularização cirúrgica do miocárdio, angioplastia ou tratamento clínico. Arq Bras Cardiol. 2007; 88(5):537-544. 
8. Garrat AM, Schmidt L, Mackintosh A, Fitzpatrick R. Quality of life measurement: bibliographic study of patient assessed health outcome measures. BMJ. 2002; 324(7351):1417-1420.

9. Green A, Liles C, Rushton A, Kyte DG. Measurement properties of patient-reported outcome measures (PROMS) in Patellofemoral Pain Syndrome: A systematic review. Man Ther 2014; 19(6):517-26.

10. Foroughi F, Sobhani S, Yoosefinejad AK, Motealleh A. Added value of isolated core postural control training on knee pain and function in women with patellofemoral pain syndrome: a randomized controlled trial. Arch Phys Med Rehabil 2018; 3(18):1-10.

11. Serbest S, Tiftikçi U, Çoban M, Çirpar M, Dağlar B. Knee pain and functional scores after intramedullary nailing of tibial shaft fractures using a suprapatellar approach. Arch Phys Med Rehabil 2018; 3(18):37-41.

12. Martinez JE, Grassi DC, Marques LG. Análise da aplicabilidade de três instrumentos de avaliação de dor em distintas unidades de atendimento: ambulatório, enfermaria e urgência. Rev Bras Reumatol 2011; 51(4):299308.

13. Kujala UM, Jaakkola LH, Koskinen SK, Taimela S, Matti H, Nelimarkka O. Scoring of patellofemoral disorders. Arthroscopy 1993; 9(2):159-63.

14. Cunha RA, Costa LO, Hespanhol JLC, Pires RS, Kujala UM, Lopes AD, et al. Translation, cross-cultural adaptation, and clinimetric testing of instruments used to assess patients with patellofemoral pain syndrome in the Brazilian population. Orthop Sports Phys Ther 2013; 43(5):332339.
15. Ciconelli RM, Ferraz MB, Santos W, Meinão I, Quaresma MR. Tradução para a lingua portuguesa e validação do questionariogenerico de avaliação de qualidade de vida SF-36. Rev Bras Reumatol 1999; 39(3):143-150.

16. Bindawas SM, Vennu V, Alfhadel S, $\mathrm{Al}$ - Otaibi AD, Binnasser AS. Knee pain and health-related quality of life among older patients with different knee osteoarthritis severity in Saudi Arabia. PLoS ONE 2018; 13(5):1-9.

17. Aquino VS, Falcon SFM, Neves LMT, Rodrigues RC, Sendín FA. Tradução e adaptação cultural para a língua portuguesa do questionário scoring of patellofemoral disorders. Acta Ortop Bras 2011; 19(5):273279.

18. Picavet HSJ, Hoeymans N. Health related quality of life in multiple musculoskeletaldiseases: SF-36 and EQ$5 \mathrm{D}$ in the DMC3 study. Ann Rheum Dis 2004; 63(6):723-729.

19. Coburn SL, Barton CJ; Filbay SR, Hart HF, Rathleff MS, Crossley KM. Quality of life in individuals with patellofemoral pain: A systematic review including meta-analysis. Phys Ther Sport 2018; 33:96-108.

20. Waiteman MC, Coura MB, Gobbi C, Briani RV, Silva DO, Azevedo FM. Comparação do nível de dor femoropatelar, atividade física e qualidade de vida entre adolescentes do sexo feminino e masculino. Rev Scientia Medica 2017; 27(1):1-6.

21. Watson CJ, Propps M, Ratner J, Zeigler DL, Horton P, Smith SS. Reliability and Responsiveness of the Lower Extremity Functional Scale and the Anterior Knee Pain Scale in Patients With Anterior Knee Pain. J Orthop Sports Phys Ther 2005; 35(3):136146. 
22. Ittenbach RF, Huang G, Barber Foss KD, Hewett TE, Myer GD. Reliability and Validity of the Anterior Knee Pain Scale: Applications for Use as an Epidemiologic Screener. PLoS One 2016; 11(7):1-6.

23. Ummels PEJ, Lenssen AF, Barendrecht M, Beurskens A JHM. Dutch translation of the Kujala Anterior Knee Pain Scale and validation in patients after knee arthroplasty. Physiother Res Int 2013; 21(11):2647-2653.

24. Silva DO, Briani RV, Ferrari D, Pazzinatto MF, Aragão FA, Azevedo FM. Q angle and subtalar pronation are not good predictors of pain and function in individuals with patellofemoral pain syndrome. Fisioter Pesq 2015; 22(3):309-316
25. Cheung RTH, Zhang Z, Ngai SPC. Different Relationships Between the Level of Patellofemoral Pain and Quality of Life in Professional and Amateur Athletes. PM\&R 2013; 5(7):568-572.

26. Pimenta FAP, Simil FF, Tôrres HOG, Amaral CFS, Rezende CF, Coelho TO, et al. Avaliação da qualidade de vida de aposentados com a utilização do questionário SF-36. Assoc Med Bras 2008; 54(1):55-60.

27. Assa T, Elbaz A, Mor A, Chechik O, Morag G, Salai M, et al. Gait metric profile of 157 patients suffering from anterior knee pain. The Knee 2013; 20(1):40-44. 